\title{
Transatlantica
}

Revue d'études américaines. American Studies Journal

2 | 2011

Sport et société / Animals and the American

Imagination

\section{Colloque " Gertrude Stein et les Arts »}

Grand Palais, Paris, 20-21 octobre 2011

\section{Emöke Simon}

\section{(2) OpenEdition}

Journals

Édition électronique

URL : https://journals.openedition.org/transatlantica/5433

DOI : 10.4000/transatlantica.5433

ISSN : 1765-2766

Éditeur

Association française d'Etudes Américaines (AFEA)

Référence électronique

Emöke Simon, « Colloque « Gertrude Stein et les Arts » », Transatlantica [En ligne], 2 | 2011, mis en ligne le 16 juin 2012, consulté le 02 février 2023. URL : http://journals.openedition.org/transatlantica/5433 ; DOI : https://doi.org/10.4000/transatlantica.5433

Ce document a été généré automatiquement le 2 février 2023.

Creative Commons - Attribution - Pas d'Utilisation Commerciale - Pas de Modification 4.0 International - CC BY-NC-ND 4.0

https://creativecommons.org/licenses/by-nc-nd/4.0/ 


\section{Colloque « Gertrude Stein et les}

\section{Arts »}

Grand Palais, Paris, 20-21 octobre 2011

\section{Emöke Simon}

1 Ce colloque a été organisé par Isabelle Alfandary et Vincent Broqua dans le cadre de l'exposition «Matisse, Cézanne, Picasso... L'aventure des Stein » (présentée au Grand Palais du 5 octobre 2011 au 16 janvier 2012) et avec le concours de la RMN (Réunion des musées nationaux), du Grand Palais, de la Terra Foundation for American Art et de l'Université Paris-Est Créteil. Le colloque a également bénéficié du soutien des services culturels de l'Ambassade des États-Unis d'Amérique.

2 L'œuvre énigmatique de Gertrude Stein s'impose comme une œuvre qui s'est nourrie des arts et qui les a nourris à son tour. À partir de ce constat, le colloque s'est donné comme objectif d'interroger la nature des rapports d'influence, de coprésence ou de superpositions qui se déplient dans les domaines des arts plastiques, du cinéma ou encore du théâtre. La proximité des tableaux au milieu desquels elle vivait et écrivait a concrétisé l'espace de réflexion du colloque en actualisant la problématique de Gertrude Stein et les arts. Comme Vincent Broqua (Université Paris-Est) le soulignait dans sa communication « Gertrude Stein traduite en art », dans un tel contexte, le nom de Gertrude Stein se charge d'une multiplicité de signifiés qui renforce davantage la nécessité de considérer l'œuvre dans un contexte élargi, à la croisée de l'histoire de l'art, de la biographie et du poétique.

\section{Gertrude Stein et « l'art interrogatif »}

3 L'influence de la peinture, plus précisément celle des tableaux de Matisse, de Cézanne et de Picasso qui enrichissent la collection de la famille Stein (Leo, Michael et sa femme Sarah, et Gertrude) à partir du Salon d'Automne de 1905, peut être retracée, selon Joan Retallack (Bard College), dans le changement de style qui s'opère au sein de l'œuvre de Stein. La révélation du Salon est celle de la possibilité d'un « art interrogatif », un art qui commence et qui continue par des questions. Dans sa communication intitulée 
«Stein and the Arts: What is the Question?", Joan Retallack soutient qu'un tel mode de fonctionnement est reconnaissable dans les textes de la collection Tender Buttons, écrits en 1912 et publiés en 1914 à New York. La rupture qui marque la différence et qui permet justement à la différence de se "répandre " (difference is spreading ", Gertrude Stein) est palpable dans la façon dont l'écriture n'est plus soumise à la rationalité descriptive mais la dépasse pour devenir elle-même vie - « living » - c'està-dire un dispositif de vitalité immédiate. Par là, cette nouvelle écriture que Stein expérimente avec Tender Buttons répond pleinement à l'appel que lance John Dewey dans Art as Experience (1934) pour un art qui est en mesure de rétablir le contact avec la matérialité vibrante du monde, mais également avec la vibration vitale de la langue. C'est ainsi que la langue peut enfin s'affirmer et acquérir un statut qui, en termes wittgensteiniens, est identifiable comme une forme de vie en elle-même.

Pour Charles Bernstein (University of Pennsylvania) et Dominique Fourcade (écrivain, critique d'art), la source de la nouvelle syntaxe steinienne est localisable dans la peinture de Matisse dans la mesure où celle-ci proposait une nouvelle composition du monde avec une nouvelle grammaire des couleurs. La grammaire qu'élabore Stein témoigne de la façon dont celle-ci a su reconnaître cette nouvelle composition, en même temps que celle de Cézanne ou de Picasso, et trouver une façon d'écrire qui soit en mesure de remettre en question la notion même de grammaire. Une proximité avec le $\mathrm{Nu}$ descendant un escalier ${ }^{1}$ (1912) est à noter selon Bernstein dans l'épaississement des aspects quadridimensionnels des mots, à travers leurs inscriptions dans un processus toujours en train d'advenir.

La communication de Marjorie Perloff (Stanford University), intitulée "A Cessation of Resemblances: Gertrude Stein's Duchamp Connection», a interrogé de près la proximité entre Marcel Duchamp et Gertrude Stein, localisable, selon elle, dans leur façon de jouer avec la langue. En 1920, Marcel Duchamp pose pour Man Ray maquillé et portant des accessoires de femme ${ }^{2}$. Son alter ego féminin ainsi créé est baptisé Rose Sélavy, une coïncidence qui ne peut pas être anodine si on considère que Duchamp avait la possibilité de connaître le poème de Stein «Sacred Emily» (écrit en 1913 et publié en 1922) dans lequel apparaît pour la première fois la fameuse «A rose is a rose is a rose is a rose ». Si l'on considère les titres des ready-made de Duchamp, la liberté ludique avec laquelle il utilise la langue est d'autant plus flagrante (par exemple L.H.O.O.Q $Q^{3}$ ). À ceci s'ajoutent des rapports de l'ordre de la collaboration artistique dans le cadre de l'exposition de Francis Picabia organisée par Léonce Rosenberg en 1932. Stein écrit un texte court pour la préface du catalogue de l'exposition de Francis Picabia (intégré par la suite dans Stanzas in Meditation), et Duchamp le traduit. Marjorie Perloff conclut que les deux artistes érotisent la langue par le même geste artistique qui consiste à décontextualiser les mots pour Stein et les objets pour Duchamp, dans le but de leur attribuer une nouvelle dimension et une nouvelle fonction. En revanche, comme l'ont souligné Charles Bernstein et Dominique Fourcade, la démarche de Gertrude Stein lui a valu une certaine marginalisation : si sa radicalité dans le domaine l'a conduite à une reconnaissance immédiate auprès de ses contemporains, en littérature elle s'est heurtée à une méfiance, voire à un rejet, dans la mesure où sa nouvelle grammaire portait atteinte "au corps même du monde» (Dominique Fourcade), c'est-à-dire à la langue. 


\section{La fabrication de Gertrude Stein par Gertrude Stein}

6 Gertrude Stein avait néanmoins tenté d'assurer la promotion de cette radicalité littéraire, comme l'a montré la communication de Wanda Corn (Stanford University), intitulée «Portraiture and the Making of Gertrude Stein ». En effet, Stein avait posé pour environ 25 artistes, aussi bien pour des peintres (Pablo Picasso, Francis Picabia, Francis Rose) que pour des photographes (Man Ray, Cecile Beaton, Carl Van Vechten). L'étude de la journaliste Corinne Gonnard («Les femmes dans l'Autobiographie d'Alice B. Toklas») a souligné également l'importance du cercle de femmes artistes qui entouraient Stein et qui ont contribué à la fabrication de son image (Nathalie Barney, Claribel et Etta Cone).

7 Wanda Corn considère donc ces portraits comme une forme moderne d'échange. D'une part ils permettaient à certains jeunes artistes d'avoir accès au monde de l'art contemporain, d'autre part ils ont ouvert un espace de construction d'une identité sur la scène artistique. Cette identité se veut multiple et soumise à un devenir qui, à partir de la posture de «New American Girl » que les premiers portraits révèlent, jusqu'à l'affirmation du "style impérial " en passant par le "type bohémien", défie les conventions de représentation du genre, du couple, mais aussi de l'acte d'écrire. Gertrude Stein n'a pas manqué de profiter de chaque occasion pour s'affirmer et s'imposer comme une figure emblématique du modernisme (comme en atteste l'un des derniers portraits réalisés par Man Ray qui la montre à côté de son portrait réalisé par Picasso), mais également comme ambassadrice des États-Unis (comme c'est le cas par exemple de la photo de Carl Van Vechten de 1935, prise après la grande tournée américaine, qui la place devant le drapeau américain).

C'est donc d'abord par le biais du portrait que Gertrude Stein marque une présence dans le monde des arts plastiques et s'impose pour beaucoup comme « The Mother of Us All ( (titre de l'opéra écrit par Stein, puis repris, entre autres par Richard Foreman). Gertrude Stein, personnage excentrique, a ouvert de multiples voies par lesquelles son écriture résonne et revit dans l'art et la littérature contemporains. Jean-Claude Lefevre, Franck David, Stan Brakhage, Carl André ne représentent qu'une infime partie de la panoplie d'artistes inspirés de ou influencés par Gertrude Stein. Comme Vincent Broqua l'a montré, l'œuvre de Carl André est un exemple adéquat de la façon dont la fameuse «A rose is a rose is a rose is a rose " peut être recyclée pour devenir matière première d'un carré sculpté sur la page.

\section{La parole steinienne - entre mouvement, image et performance}

Objet à recycler, à ouvrir comme une boîte ou à transformer, la parole steinienne est aussi présente sur la scène théâtrale depuis 1934 - date de la première représentation de l'opéra Fours Saints in Three Acts à Hartford (musique composée par Virgil Thomson). Dans une communication intitulée "Théâtre et théâtralité de Gertrude Stein », MarieClaire Pasquier (Université Paris-Ouest) a retracé la manière dont le théâtre postmoderne s'est emparé du texte steinien chez Al Carmines ou Richard Foreman par exemple. La disponibilité à l'espace scénique du texte steinien, comme Thalia Field (écrivaine) l'a suggéré lors de la table ronde qui réunissait, entre autres, Marie-Claire 
Pasquier, Alexis Forestier (metteur en scène de Docteur Faustus ou la fête électrique), Cécile Saint-Paul (actrice), ne fait que renforcer la manière dont la parole steinienne est déjà un objet de performance sur la scène de la page avec un souci plastique digne d'un metteur en scène. Cette performance se double d'une autre qui, elle, peut être conditionnée par un processus d'obscurcissement: comme l'indique Antoine Cazé (Université Paris-Diderot) dans sa communication intitulée "Stein : Méditations sans images ", il s'agit d'opacifier la vue pour voir et d'ouvrir ainsi un espace qui soit un milieu dans la langue même. Afin d'être saisissable, cette façon de "monter dans le langage pour faire stationner la langue » (Antoine Cazé) nécessite un changement de perspective - le rapport langue-objet, au lieu d'être soumis à un résultat valide, vérifiable par l'adéquation mot/image doit être perçu dans le flux même du processus par lequel le mot pointe l'objet.

La modernité de la perception qui caractérise la composition steinienne est également saisissable dans sa proximité avec le cinéma. La communication d'Isabelle Alfandary (Université Paris-Est) intitulée " Gertrude Stein et Eadweard Muybridge » exploitait le " cinématographe intérieur » chez Stein, c'est-à-dire les points de rencontres entre l'image précinématographique et la technique d'écriture steinienne. Nourrie par la tension entre l'immobile et le mobile, les variations d'intensité de l'excitation, l'obsession du présent, l'écriture de Gertrude Stein donne à voir le rythme, un "voir sonore ", qui, à la manière des images de Muybridge, est susceptible de rendre visible le devenir-imperceptible du temps. Les propos d'Abigail Lang (Université Paris-Diderot) au sujet de "Stein cinématographe " suggèrent que le devenir-imperceptible est également localisable dans la façon dont le cinéma exprime ce que Stein désignait par l'expression " the quality of being disembodied ", c'est-à-dire une présence absente que l'écran cinématographique assure aux personnages. C'est ainsi que Stein définissait la spécificité américaine, mais c'est aussi une manière d'être de l'image cinématographique que Stein semblait maîtriser parfaitement, comme en témoigne le texte « A Movie » destiné à être le script d'un film. L'analyse d'Abigail Lang de ce texte a permis également d'interroger la manière dont les techniques cinématographiques telles que le zoom sont intégrées dans l'écriture de Stein.

11 La réflexion personnelle de Jean-Marie Gleize (écrivain; ENS Lyon), doublée de la lecture d'un extrait d'Everybody's Autobiography (1937) - récit d'une ronde de nuit à Chicago dans une voiture de police - dans le cadre de la soirée de lecture dédiée à Stein $^{4}$, met en évidence la manière dont elle célébrait le mouvement avec une passion pour l'immobilité, les deux dimensions étant deux formes d'être au présent. Par là, selon Jean-Marie Gleize, Gertrude Stein propose une pratique expérimentale de la vie et lance un appel à veiller "pour être présent au présent ", sans pathos, sans action. Vivre et écrire se superposent comme deux dimensions de la même pratique, d'une œuvre qui demeure une "œuvre à venir ", dans «l'assomption de l'obscurité comme lumière » (Jean-Marie Gleize).

12 Organisé autour de l'idée du dialogue, le colloque a réaffirmé le dynamisme et la plasticité de l'écriture steinienne, toujours prête à se laisser investir par le lecteur et le transformer en partenaire actif de la fabrication du texte. Mais ce colloque lui a surtout assuré une présence qui a su déborder les frontières du monde universitaire. De nouveaux espaces de résonances de la parole steinienne ont été ainsi ouverts.

Lien vers le programme du colloque: http://www.rmn.fr/activites-culturellesgratuites-2509. 


\section{NOTES}

1. http://www.marcelduchamp.net/news.php?id=701

2. http://www.artandculture.com/media/show?media_id=70588\&media_type=Image

3.

http://www.centrepompidou.fr/Pompidou/Manifs.nsf/0/ F6B2462E8A10AD95C125704500318AD9?OpenDocument

4. Les lectures bilingues de Charles Bernstein, de Joan Retallack, de Thalia Field, d'Abigail Lang ou encore de Martin Richet, ont également ouvert de nouvelles perspectives de lecture ou d'approche du texte steinien.

INDEX

Thèmes : Actualité de la recherche

\section{AUTEUR}

\section{EMÖKE SIMON}

Université Lumière Lyon 2 